\title{
Texture Guided Active Appearance Model Propagation for Prostate Segmentation
}

\author{
Soumya Ghose ${ }^{1,2}$, Arnau Oliver ${ }^{1}$, Robert Martí ${ }^{1}$, Xavier Lladó ${ }^{1}$, \\ Jordi Freixenet $^{1}$, Joan C. Vilanova ${ }^{3}$, and Fabrice Meriaudeau ${ }^{2}$ \\ ${ }^{1}$ Computer Vision and Robotics Group, University of Girona, Campus Montilivi, \\ Edifici P-IV, Av. Lluís Santaló , s/n, 17071 Girona, Spain \\ ${ }^{2}$ Laboratoire Le2I - UMR CNRS 5158, Université de Bourgogne, \\ 12 Rue de la Fonderie, 71200 Le Creusot, France \\ ${ }^{3}$ Girona Magnetic Resonance Imaging Center, Girona, Spain
}

\begin{abstract}
Fusion of Magnetic Resonance Imaging (MRI) and Trans Rectal Ultra Sound (TRUS) images during TRUS guided prostate biopsy improves localization of the malignant tissues. Segmented prostate in TRUS and MRI improve registration accuracy and reduce computational cost of the procedure. However, accurate segmentation of the prostate in TRUS images can be a challenging task due to low signal to noise ratio, heterogeneous intensity distribution inside the prostate, and imaging artifacts like speckle noise and shadow. We propose to use texture features from approximation coefficients of Haar wavelet transform for propagation of a shape and appearance based statistical model to segment the prostate in a multi-resolution framework. A parametric model of the propagating contour is derived from Principal Component Analysis of prior shape and texture informations of the prostate from the training data. The parameters are then modified with prior knowledge of the optimization space to achieve optimal prostate segmentation. The proposed method achieves a mean Dice Similarity Coefficient value of $0.95 \pm 0.01$, and mean segmentation time of $0.72 \pm 0.05$ seconds when validated on 25 TRUS images, grabbed from video sequences, in a leaveone-out validation framework. Our proposed model performs computationally efficient accurate prostate segmentation in presence of intensity heterogeneity and imaging artifacts.
\end{abstract}

\section{Introduction}

Prostate cancer is a major health problem with more than 670,000 people being diagnosed every year worldwide [1. In clinical practice TRUS guided needle biopsy is performed to diagnose prostate cancer, due to the real time nature of the imaging system, ease of use, and portability. However, TRUS images have low signal to noise ratio (SNR) and detection of malignant tissues in TRUS images is difficult. MR images provide higher contrast for soft tissues of the prostate that allows a better detection of cancerous tissues. However, interventional MRI

A. Madabhushi et al. (Eds.): Prostate Cancer Imaging 2010, LNCS 6367, pp. 111-120, 2010.

(C) Springer-Verlag Berlin Heidelberg 2010 
guided biopsy is expensive and complicated. Therefore, one solution lies in the fusion of the two imaging modalities to exploit the high quality of MR images in TRUS interventional biopsies. Real-time registration between two dimensional (2D) TRUS video sequence and three dimensional (3D) MR images is necessary for such a process. Registration performed on accurately segmented contour of TRUS and MR images will aid in designing computationally efficient and accurate registration procedures [20. Thus, real-time accurate $2 \mathrm{D}$ segmentation of TRUS images from TRUS video sequence is necessary for the process.

Prostate segmentation in TRUS images is particularly challenging. Low SNR in a TRUS image of prostate reduces the accuracy of intensity based segmentation algorithms. Approaches working on traditional edge detection filters like Sobel, Prewitt are adversely affected with the issues of high noise and low SNR producing discontinuous prostate edges. Heterogeneous intensity distribution inside the prostate is a hindrance in designing a global descriptor. Added to these, shadow artifacts, speckle noise and micro calcification significantly challenges the segmentation of the prostate.

Incorporating prior shape and intensity informations in the segmentation methods improve the prostate segmentation accuracy. In 1998 Cootes et al. [6] provided an efficient framework for combining shape and intensity prior in their Active Appearance Model (AAM). Medina et al. [16] used AAM to segment prostate in Two Dimensional (2D) TRUS images with an overlap ratio of $96 \%$. However, it is argued by Wolstenholme and Taylor [19] that the time complexity involved with AAM is high and is unsuitable for real time procedures. Instead they proposed to use wavelet coefficients of training images for building the AAM. Larsen et al. 14 showed that frequency separation in wavelet transform allowed an edge enhancement that provided better result in terms of segmentation accuracy compared to traditional AAMs. They proposed to use a texture vector comprising the truncated detail and approximation coefficients in multiresolution framework.

To address the challenges involved with prostate segmentation in 2D TRUS images, we propose a novel AAM that is propagated by the approximation coefficients of Haar wavelet transform in a multi-resolution framework . Compared to the use of intensity as in traditional AAM [6], the use of approximation coefficients of the wavelet transformed image improves the computational time and accuracy of prostate segmentation. The approach is similar to Larsen et al. [14], while deviating from their model of using both detail and approximation coefficients to construct a texture vector for AAM propagation we propose to use the approximation coefficients of the Haar wavelet transformed image. The performance of our method is validated using 25 images captured from TRUS video sequence. Experimental results show that our method is unaffected by low SNR, intensity heterogeneities and micro calcifications inside prostate region and imaging artifacts like shadow and speckle noise.

The rest of the paper is organized as follows. The texture driven AAM is formulated in Section 2 followed by quantitative and qualitative evaluation of our method in Section 3. We finally draw conclusion in Section 4. 


\section{Methods}

The proposed method is developed on two major components: the adaptation of AAM and incorporation of texture information. Traditional AAM is presented first followed by a comprehensive discussion of using Haar wavelet in extraction of texture feature to build the model. Finally, the model building and propagation procedure are presented.

\subsection{Active Appearance Model}

AAM provides a compact parametric framework utilizing prior shape and intensity variabilities learned from a training model to segment an unseen test image exploiting the prior knowledge of the nature of the optimization space [14]. The process of building AAM may be partitioned into two separate tasks; building the shape model from the contours and building the appearance model from the intensity distribution inside the manually segmented region. Finally, the two models are combined to produce AAM that incorporates prior knowledge of shape and intensity variabilities.

Generalized Procrustes Analysis (GPA) of the Point Distribution Model (PDM) 5] built from manually segmented contours is used to align the PDM. Principal Component Analysis (PCA) of the aligned PDMs are used to identify the principal components of the variations in shape and suppress redundancy. Intensity distribution are warped into correspondence using a piece wise affine warp and sampled from shape free reference. PCA of the intensity distribution is used to identify the principal components of intensity variations.

The model may be formalized in the following manner. In eq. 2 let $E\{s\}$ and $E\{t\}$ represent the shape and intensity models where $s$ and $t$ are the shape and the intensities of the corresponding training images, $\bar{s}$ and $\bar{t}$ denote the mean shape and intensity respectively, then $\Phi_{s}$ and $\Phi_{t}$ contain the first $p$ eigenvectors of the estimated joint dispersion matrix of shape and intensity and $\theta$ represents the corresponding eigenvalues.

$$
\begin{aligned}
E\{s\} & =\bar{s}+\Phi_{s} \theta \\
E\{t\} & =\bar{t}+\Phi_{t} \theta
\end{aligned}
$$

In addition to the parameters $\theta$, four parameters, two translations, rotation and scale are represented by $\psi$. In order to infer the parameters of $\theta$ and $\psi$ of a previously unseen image, a Gaussian error model between model and pixel intensities is assumed [14]. Furthermore, a linear relationship between changes in parameters and difference between model and image pixel intensities $\Delta t$ is assumed as shown in eq, 2

$$
\Delta t=X\left[\begin{array}{c}
\Delta \psi \\
\Delta \theta
\end{array}\right]
$$




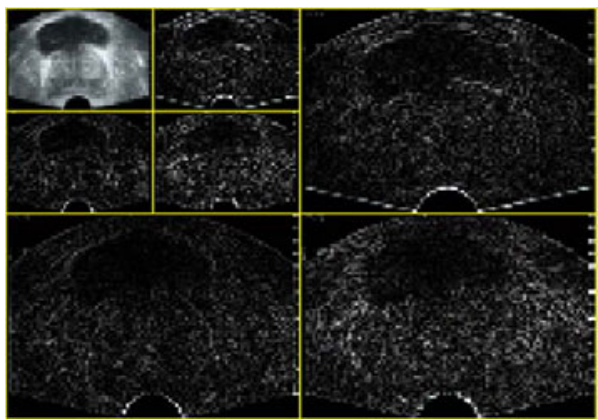

Fig. 1. Second level Haar wavelet decomposition of the prostate

$X$ is estimated from weighted averaging over perturbation of model parameters and training examples. Eq,2 is solved in least square manner fitting error as shown in eq 3

$$
\left[\begin{array}{l}
\widehat{\psi} \\
\widehat{\theta}
\end{array}\right]=\left(X^{T} X\right)^{-1} X^{T} \delta t
$$

The problem is computationally expensive. To reduce the computational time we propose to use approximation coefficients of Haar wavelet transform. However, this will introduce the additional time requirement of transformation of the image into a new representation. Since the transformation is based on sparse matrix, the computational burden can be considerably reduced as stated in [14].

\subsection{Texture Analysis Using Haar Wavelets}

Wavelets are a family of basis functions that decomposes signal into frequency and time domains. In practice, a set of linear, rank preserving matrix operations are carried out in a convolution scheme to decompose an image by a high pass filter and by a low pass filter into different sub-bands. For a 2D image the high pass filter generates three detail coefficient sub-bands corresponding to horizontal, vertical and the diagonal edges. The approximation sub band obtained from low pass filter, is down-sampled and is further decomposed to analyze the detail and the approximation coefficients at a coarser resolution. The Haar wavelet decomposition of a 2D TRUS image of the prostate is shown in fig.1

The property of the wavelets that allows to analyze the detail and the approximation coefficients in a multi-resolution framework proves to be a powerful tool for edge and texture analysis [17. To introduce wavelet coefficients in AAM, we formalize the framework with the used notation. First, let a n-level wavelet transform be denoted by

$$
\widehat{w}=\left[\widehat{a}^{T} \widehat{u}_{1}^{T} \ldots \widehat{u}_{n}^{T}\right]^{T}
$$


where, $\widehat{a}$ and $\widehat{u}$ represent the approximation and the detail coefficients respectively, and $\widehat{w}$ is the wavelet transformed image [14]. The detail coefficients are suppressed to produce a truncated wavelet basis as

$$
b(\widehat{w})=C \widehat{w}=\left[\widehat{a}^{T} 0 \ldots 0\right]^{T}
$$

where, $C$ corresponds to a modified identity matrix with the rows corresponding to the detail coefficients removed. The AAM is built on the truncated wavelet basis constituting the texture. The PCA of the texture is given by 6

$$
a=\bar{a}+\Phi_{a} B_{w}
$$

where $\bar{a}$ is the mean of the approximation coefficients, $\Phi_{a}$ and $B_{w}$ are the matrices constituting the eigenvectors and their corresponding eigenvalue respectively, that represent the principal components of the approximation coefficients.

Suppressing the high frequency components certainly reduces texture information. However, the texture information that are to be preserved is context dependent. To ensure the uniformity of texture inside the prostate, suppression of the detail coefficients is desirable since, the high gradient energies are minimized. Moreover, speckle noise and micro calcifications, the high frequency components, inside the prostate tissues are considerably reduced by the suppression of the detail coefficients, producing appropriate texture map. Finally, such suppression reduces the computational complexities involved with the fitting of a new image to the model. It is to be noted that, significant texture informations are preserved in the high energy components that are the approximation coefficients 17. PCA of the approximation coefficients helps us to suppress noise in the underlying texture by preserving the important components only.

\subsection{Model Building}

The model building procedure could be summarized in the following steps,

1. Automatic creation of PDM from the segmented contour using a radial search method.

2. All the PDMs are aligned using GPA.

3. PCA of the aligned contours is done to identify the principal components of the shape variation.

4. Intensities are sampled from each of the aligned PDM.

5. Wavelet transform of the intensities produce the approximation and the detail coefficients.

6. The detail coefficients are suppressed and the approximation coefficients are then used to identify the principle variations of the texture which is used to build the AAM.

Larsen et al. 14 claimed that wavelet decomposition of an image in multiresolution propagates fitting error due to loss of texture information. Therefore, we have adopted wavelet decomposition of the first level and subsequently fitted 


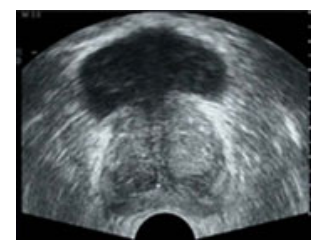

(a)

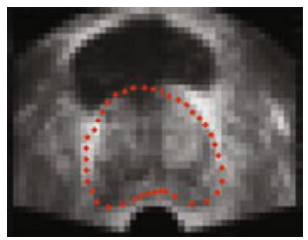

(d)

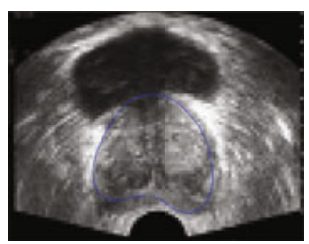

(b)

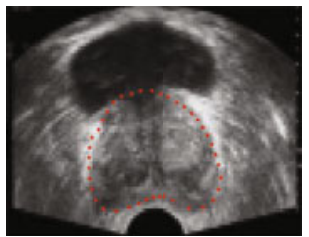

(e)

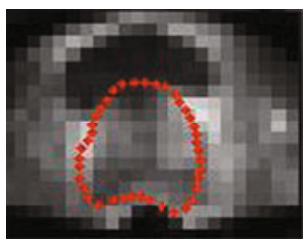

(c)

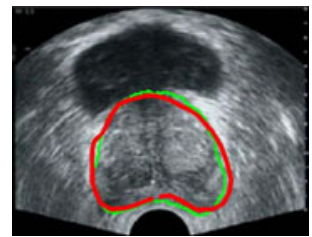

(f)

Fig. 2. Multi-resolution functioning of the model (a) 2D TRUS image of the prostate, (b) Manual initialization of the mean model (blue contour) by clicking on the center of the prostate, (c) Level 4 segmentation result, (d)Level 3 segmentation result, (e) Level 2 segmentation result, (f) Final segmentation result. Manual segmentation shown with green contour and the red contour show the segmentation achieved.

our model to the approximation coefficients in coarser to finer spatial resolutions to reduce texture dependent fitting error. Multi-resolution fitting of an image improves segmentation accuracy. The multi-resolution functioning of the model is illustrated in fig 2 .

It is to be noted that the mean model is initialized by clicking in any position close to the center of the prostate decided on visual inspection. The mean model initialization and subsequent multi-resolution segmentations are produced based on the approximation coefficients of the Haar wavelet.

\section{Experimental Results}

We have validated the accuracy and robustness of our approach on a series of 25 prostate ultrasound images using leave-one-out evaluation strategy. The images of resolution $538 \times 418$ are grabbed from TRUS video sequences (acquired with a Siemens Aquson). Our method was implemented in Matlab 7 on a Intel Core 2 Duo T5250 processor of $1.5 \mathrm{Ghz}$ processor speed and 2 GB RAM. We have used most of the popular prostate segmentation evaluation metrics in order to evaluate our approach. The average values for all the 25 images show, Dice similarity coefficient DSC value of $0.95 \pm 0.01,95 \%$ Hausdorff Distance (HD) of $5.08 \pm 1.18 \mathrm{~mm}$, Mean Absolute Distance (MAD) of $1.48 \pm 0.36 \mathrm{~mm}$, Maximum distance (MaxD) of $5.01 \pm 1.13 \mathrm{~mm}$, specificity of $0.92 \pm 0.02$ and sensitivity value of $0.998 \pm 0.001$ with a mean segmentation time of $0.72 \pm 0.05$ seconds.

For a qualitative analysis of our method we have presented the 25 figures used for validation with green contours depicting the ground truth and the red contours indicating the segmented prostates as shown in fig 3 . 

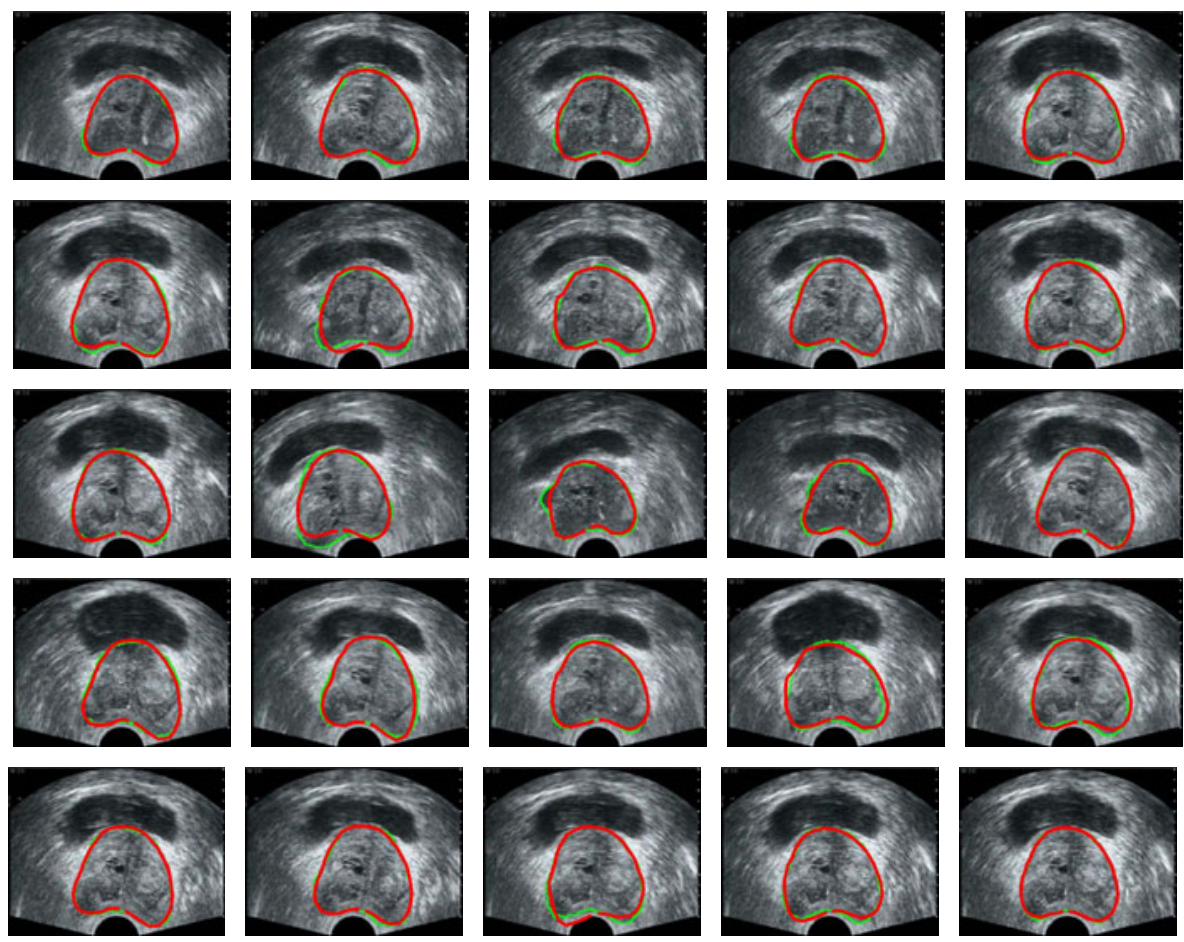

Fig. 3. Qualitative segmentation results. The green contour gives the manual segmentation and the red contour gives the obtained result.

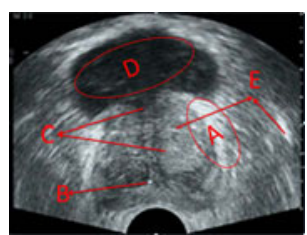

(a)

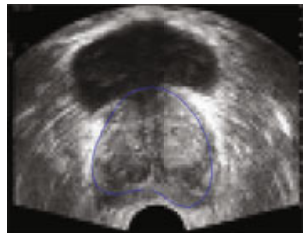

(b)

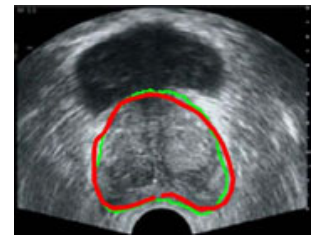

(c)

Fig. 4. (a) Prostate artifacts in TRUS image of the prostate, $\mathrm{A}=$ Low SNR, B=MicroCalcification, $\mathrm{C}=$ Intensity difference inside prostate region, $\mathrm{D}=$ Shadow Artifacts, $\mathrm{E}=$ Speckle Noise. (b) Manual initialization of the mean model (blue contour) by clicking on prostate center, (c) Final segmentation result. Manual segmentation shown with green contour and the red contour show the segmentation achieved.

As stated before in Section [1 the robustness of the the proposed method against low SNR, intensity heterogeneities, shadow artifacts, speckle noise and micro calcification inside prostate is demonstrated in fig 4 As seen in fig 4(b) that on initialization a section of the mean model (blue contour) is located in a region of shadow artifact, the model successfully avoids the artifact and segments the prostate accurately with a DSC value of 0.94 . 
The mean model initialization in the TRUS images is done on visual inspection and therefore varies from one case to the other. Low standard deviation values of $0.01,0.015$ and 0.0001 associated with the DSC, specificity and sensitivity values seems to indicate that the final segmentation result of our method is not affected by the manual initialization of the mean model. To further validate our claim the standard deviation value of mean DSC over four independent test was computed and the mean of these values was 0.014. This further proves that accuracy of the process is indifferent to manual intialization. However, the mean model could be initialized automatically on the assumption that the prostate is visible in the center of the TRUS image. A more sophisticated approach would be an initial rough classification of the TRUS image to identify the prostate region and then initialize the mean model at the center of the prostate region.

Comparison of different prostate segmentation methodologies is difficult in absence of public datasets and standardized evaluation metrics, since the methods are developed with a wide variety of algorithms and with specific application requirements. However, to have an overall qualitative estimate of the functioning of our method we observe that the mean segmentation time of $0.72 \pm 0.05$ seconds for an image is comparable to [2] (less than a second), 15](2.1 second), 11] (5 seconds), 3 ( 5 seconds) and inferior only to 20. that achieves segmentation time of $0.3 \mathrm{sec}$ in $\mathrm{C}++$ and ITK framework. To have an estimate of overlap accuracy DSC value of $0.95 \pm 0.01$ is comparable to different measure of overlap accuracy value obtained by 12 (Area difference 8.48\%), 8 (Area difference $4.79 \pm 0.68 \%$ ), 10] (Average similarity 89\%), 18 (Area overlap error 3.98 $\pm 0.97 \%$ ), 3] (Area overlap $93 \pm 0.9 \%)$, [16] (Area overlap 93\%), 21](Area overlap 91\%) and [4](Area accuracy $94.05 \%)$. MAD of our method of $1.48 \pm 0.36 \mathrm{~mm}$ is comparable to 20] (MAD $1.79 \pm 0.95 \mathrm{~mm})$, [12](MAD $2.61 \mathrm{~mm})$, [13] (MAD $4.4 \pm 1.8$ pixels), 9] (MAD $2.79 \pm 1.94 \mathrm{~mm})$, [7] $(6.21 \pm 4.03 \mathrm{~mm})$, and [1] (Contour average distance $1.36 \pm 0.6 \mathrm{~mm})$. With reasonable conviction we can state that qualitatively our method performs well compared to some of the works in literature.

\section{Conclusion}

A novel approach of using Haar wavelet approximation coefficients to propagate AAMs with the goal of segmenting the prostate in 2D TRUS images have been proposed. Our approach is accurate, computationally efficient and robust to low SNR, intensity heterogeneity of prostate tisssue, shadow artifacts, speckle noise and micro calcification. It is observed that the use of the Haar wavelet approximation coefficients only, does not deteriorate the segmentation accuracy. While the proposed method is validated with prostate mid-gland images the effectiveness of the method against base and apical region slices is yet to be validated. Computational time of the process is fast but not suitable for realtime applications like MRI-TRUS fusion. We would like to explore the possibility of using the CUDA platform to achieve necessary hardware acceleration and parallelization in order to produce real time $2 \mathrm{D}$ segmentation of the prostate in TRUS images. 


\section{Acknowledgements}

This research has been funded by VALTEC 08-1-0039 of Generalitat de Catalunya, Spain and Conseil Régional de Bourgogne, France.

\section{References}

1. Prostate Cancer Statistics - Key Facts (2009), http://info.cancerresearchuk.org/cancerstats/types/prostate

2. Abolmaesumi, P., Sirouspour, M.: Segmentation of Prostate Contours from Ultrasound Images. In: IEEE International Conference on Acoustics, Speech, and Signal Processing, vol. 3, pp. 517-520 (2004)

3. Betrouni, N., Vermandel, M., Pasquier, D., Maouche, S., Rousseau, J.: Segmentation of Abdominal Ultrasound Images of the Prostate Using A priori Information and an Adapted Noise Filter. Computerized Medical Imaging and Graphics 29, 43-51 (2005)

4. Chang, C.Y., Wu, Y.L., Tsai, Y.S.: Integrating the Validation Incremental Neural Network and Radial-Basis Function Neural Network for Segmenting Prostate in Ultrasound Images. In: Proceedings of the 9th International Conference on Hybrid Intelligent Systems, vol. 1, pp. 198-203 (2009)

5. Cootes, T.F., Hill, A., Taylor, C.J., Haslam, J.: The Use of Active Shape Model for Locating Structures in Medical Images. Image and Vision Computing 12, 355-366 (1994)

6. Cootes, T.F., Edwards, G., Taylor, C.: Active Appearance Models. In: Burkhardt, H., Neumann, B. (eds.) ECCV 1998. LNCS, vol. 1407, pp. 484-498. Springer, Heidelberg (1998)

7. Cosío, F.A., Davies, B.L.: Automated Prostate Recognition: A Key Process for Clinically Effective Robotic Prostatectomy. Medical and Biological Engineering and Computing 37, 236-243 (1999)

8. Ding, M., Chen, C., Wang, Y., Gyacskov, I., Fenster, A.: Prostate Segmentation in 3D US Images Using the Cardinal-Spline Based Discrete Dynamic Contour. In: Proceedings of SPIE Medical Imaging: Visualization, Image-Guided Procedures, and Display, vol. 5029, pp. 69-76 (2003)

9. Ding, M., Gyacskov, I., Yuan, X., Drangova, M., Downey, D., Fenster, A.: SliceBased Prostate Segmentation in 3D US Images Using Continuity Constraint. In: 27th Annual International Conference of the Engineering in Medicine and Biology Society, pp. 662-665 (2006)

10. Ghanei, A., Soltanian-Zadeh, H., Ratkewicz, A., Yin, F.F.: A Three-Dimensional Deformable Model for Segmentation of Human Prostate from Ultrasound Images. Medical Physics 28, 2147-2153 (2001)

11. Gong, L., Pathak, S.D., Haynor, D.R., Cho, P.S., Kim, Y.: Parametric Shape Modeling Using Deformable Superellipses for Prostate Segmentation. IEEE Transactions on Medical Imaging 23, 340-349 (2004)

12. Knoll, C., Alcañiz, M., Monserrat, C., Grau, V., Juan, M.C.: Outlining of the Prostate Using Snakes with Shape Restrictions Based on the Wavelet Transform (doctoral thesis: Dissertation). Pattern Recognition 32, 1767-1781 (1999)

13. Ladak, H.M., Mao, F., Wang, Y., Downey, D.B., Steinman, D.A., Fenster, A.: Prostate Segmentation from 2D Ultrasound Images. In: Proceedings of the 22nd Annual International Conference of the IEEE Engineering in Medicine and Biology Society, vol. 4, pp. 3188-3191 (2000) 
14. Larsen, R., Stegmann, M.B., Darkner, S., Forchhammer, S., Cootes, T.F., Ersbll, B.K.: Texture Enhanced Appearance Models. Computer Vision and Image Understanding 106, 20-30 (2007)

15. Liu, Y.J., Ng, W.S., Teo, M.Y., Lim, H.C.: Computerised Prostate Boundary Estimation of Ultrasound Images Using Radial Bas-Relief Method. Medical and Biology Engineering and Computing 35, 445-454 (1997)

16. Medina, R., Bravo, A., Windyga, P., Toro, J., Yan, P., Onik, G.: A 2D Active Appearance Model For Prostate Segmentation in Ultrasound Images. In: 27th Annual International Conference of the IEEE Engineering in Medicine and Biology Society, pp. 3363-3366 (2005)

17. Petrou, M., Sevilla, P.G.: Image Processing: Dealing With Texture, 1st edn. Wiley, Chichester (2006)

18. Shen, D., Zhan, Y., Davatzikos, C.: Segmentation of Prostate Boundaries from Ultrasound Images Using Statistical Shape Model. IEEE Transactions on Medical Imaging 22, 539-551 (2003)

19. Wolstenholme, C.B.H., Taylor, C.J.: Wavelet Compression of Active Appearance Models. In: Taylor, C., Colchester, A. (eds.) MICCAI 1999. LNCS, vol. 1679, pp. 544-554. Springer, Heidelberg (1999)

20. Yan, P., Xu, S., Turkbey, B., Kruecker, J.: Optimal Search Guided by Partial Active Shape Model for Prostate Segmentation in TRUS Images. In: Proceedings of the SPIE Medical Imaging: Visualization, Image-Guided Procedures, and Modeling, vol. 7261, pp. 72611G-72611G-11 (2009)

21. Zaim, A.: Automatic Segmentation of the Prostate from Ultrasound Data Using Feature-Based Self Organizing Map. In: Kalviainen, H., Parkkinen, J., Kaarna, A. (eds.) SCIA 2005. LNCS, vol. 3540, pp. 1259-1265. Springer, Heidelberg (2005) 